\title{
The Model of Collaborative Learning GRID to Activate Interactivity for Knowledge Building
}

\author{
Toshio OKAMOTO* \& Mizue KAYAMA** \\ *University of Electro- Communications \\ Graduate School of Information Systems \\ Chofugaoka 1-5-1, Chofu, Tokyo 182-8585, JAPAN \\ Tel : +81424435620/ Fax : +81424896070 \\ okamoto@ai.is.uec.ac.jp \\ **Senshu University \\ School of Network and Information \\ Higashimita 2-1-1, Tama, Kawasaki, Kanagawa 214-8580, \\ JAPAN \\ Tel \& Fax : +81 449110570 \\ kayama@isc.senshu-u.ac.jp
} Keywords : $\quad$ Collaborative Learning
Collaborative Memory, Social Computing

\begin{abstract}
The purpose of this study is to support the learning activity in the Internet learning space. In this paper, we examine the GRID technology as the knowledge management for supporting "collaborative learning" / "collaboration in leaming" (CL). RAPSODY-EX (REX) is a distributed learning support environment organized as a learning infrastructure. REX can effectively carry out to support collaborative activities in asynchronous/synchronous leaming mode. The mixed distributed leaming environment is utilized as a new learning ecology, where individual / collaborative leaming environment with audio/visual channel (ike as a videoconference) are performed on the multimedia communication network. In this mixed distributed leaming environment, people can arrange, modify and integrate educational information for the purpose of investigating, decision making, planning, problem solving, building knowledge and self development. Diverse information in the educational context is referred and reused as knowledge which oneself and others can practically utilize. We aim at constructing the growing digital portfolio database for learning knowledge management in Internet environment. In addition, we explore the GRID technology of activating human-interactivity for knowledgemining/discovering.
\end{abstract}

\section{Introduction}

The society is changing along with the explosive growth of Internet. This growth is so closely, tightly, and widely that everyone even feels the power of the information evolution. Education certainly is riding on the waves. Internet is becoming the catchphrase in the world of school education, which makes the distance education possible to anybody at anytime and from anywhere. As such, a new learning style 'e-Learning' emerged under the umbrella new concept of 'Learning Ecology and Pedagogy", where Internet raises the level of communications and collaborations among people via technology.

Nowadays, the word/system of "e-Learning" is rapidly spreading out according to popularization of Internet. As for advantages of Internet, people can communicate each other for anyone, anytime and anywhere. Moreover people can share, rebuild, stock and reuse the various kind of information. Here, it seems that the concept of "e-Learning" gets the citizenship in the society instead of CAI (Computer Assisted Instruction). Along with this stream/trend, we recognize the necessity of construction to new learning society such as learning individuals, learning organization and learning community. Above mentioned, we can say that Internet is a kind of "Treasure Island" of educational resources from the world wide stance, though it includes much harmful information.

In asynchronous learning, the transformer of knowledge and the transformee of knowledge communicate with a time lag. In such a situation, more positive support is required to realize an effective and efficient learning activity. We need to build a learning infrastructure with learning spaces with various functions.

Recently, the thought of GRID technology in e-Learning are introduced, which means conceptual and technical aspects of electronic learning. Furthermore, this concept is being extended toward knowledge GRID, GRID Intelligence, and distributed artificial intelligence for effective knowledge communication/ building/management. We investigate the mechanism of transmission and management of knowledge for the development of the knowledge community in the learning space, within the educational context. In this paper, we discuss about the technology the knowledge management and the knowledge representation of the learning information for the CL support in consideration of GRID technology under the context of e- Learning.

\section{Collaboration in Learning 2.1 Some Definitions}

In terms of Roschelle \& Teasley (1995), they defined "collaboration" to be "a coordinated, synchronous activity that is the result of a continued attempt to construct and maintain a shared conception of a problem". Dillenbourg(1999) takes up the following 4 points as the features of $\mathrm{CL}$, which are a situation, interactions, process(learning mechanism) and effects. Cowie \& Rudduck(1988) defined that collaboration in leaning is the opportunity to learn through the expression and exploration of diverse ideas and experiences in cooperative company. It is not about competing with fellow members of the group and winning, but using the diverse resources available in the group to deepen understanding, sharpen judgment and extend knowledge".

In consideration of those views, Okamoto (2000) pointed out that CL should emphasize as follows:

1) Process/situated context

2) Individual learning achievement such as knowledge acquisition, skill formation and concept formation, learning set

3) Versatile cognition for both of holistic and serialistic thinking schema

4) Understandings of objective relationship among self/you/he or she

5) Effects of observation learning (reflection/self-monitoring)

CL doesn't depend on place and time. Especially, In Internet environment, the type of asynchronous ecology of $\mathrm{CL}$ is more useful rather than the synchronous one (such as a Videoconference). Moreover, in the process of CL, individual learning may be sometime embedded based on a certain curriculum in schools and vise versa. Collaboration in learning and/or collaborative learning often happens during ordinal educational situation. In this study, CL is thought as a fundamental and essential learning activity. 
Table 1: Activities on Collaborative Leaming

\begin{tabular}{|c|c|}
\hline [Activity-cognitive level] & [Activity-social level] \\
\hline - Discussing & - Observing/Suggesting \\
\hline -Planing/Designing & - Role-taking/Cooperating \\
\hline - Data/Idea sharing & - Coordinaing / Controling \\
\hline -Evaluating/Finding solution & - Social interacting \\
\hline - Building knowledge & - Facilitating / Supervising \\
\hline
\end{tabular}

2.2 Technological/Functional Conditions to Encourage CL

In general, we can divide the activities on collaborative learning into two classes. The social activities and the cognitive activities are estimated. Table 1 shows these classes. Based on these activities, the resources required in CL environment are taken up as follows:

- Technologically mediated dialogue channel

- Shared workplace for a group

- Personal workplace

- Learning materials/ learning tools

- Analyzing tools ofData/Information

- Repository/Memory for data/information revealed in CL

- Reference channel for the collaborative repository

- Modeling tools for monitoring the process ofCL

To encourage the CL, we implemented the RAPSODYEX.(REX) REX is a learning framework with above resources. This system can store all leaning activities log in the digital portfolio holder and participants can review those data in order to diagnose/evaluate their achievements. Also, the participants can refer the flow/stock of knowledge for an arbitrary situation of collaborative learning. The repository-module in the REX can work to analyze/manage knowledge that each of participants revealed/found in the process of collaborative learning.

\section{The Schema and Functions of Learning GRID}

In this study, the information/knowledge processing is considered as a process of the knowledge management in the learning context. Knowledge management is defined like follows (Davenport 1997). The knowledge management is "the systematic process of finding, selecting, organizing, distilling and presenting information in a way that improves an employee's comprehension in a specific area of interest."

Here, we propose the functions of Learning GRID as the knowledge management between learning environments (like as a set of the REX). It guarantees the mutual interactivity among learners and activates knowledge building. In some sense, GRID means the role of exchanging knowledge or artifacts in a set of marketplaces that can stock the various kind of information. At the same time, learners need the value of sense with sharing/re-using manners. We have developed GRID technology as knowledge mining in collaborative learning process. REX plays as a marketplace in the GRID world.

Figure 1 shows the meaning of Leaming GRID which plays roles of the set of the marketplaces for knowledge transmission, transformation and exchange. Moreover, the function of Learning GRID is in charge of bring about building and discover of knowledge through assimilation/ accommodation, differentiating/ integrating occurred in collaborative learning. We aim to realize this mechanism as social computing by active collaborative memory (CM).

Learning GRID we intend plays roles of encouraging collaborative learning. Figure 2 shows the framework of learning GRID technology. From participants' performance information and learning resource information. Learning GRID has the functions of modeling the situation of collaborative learning and perturbing a learner's behavior for knowledge exchanging, transforming and

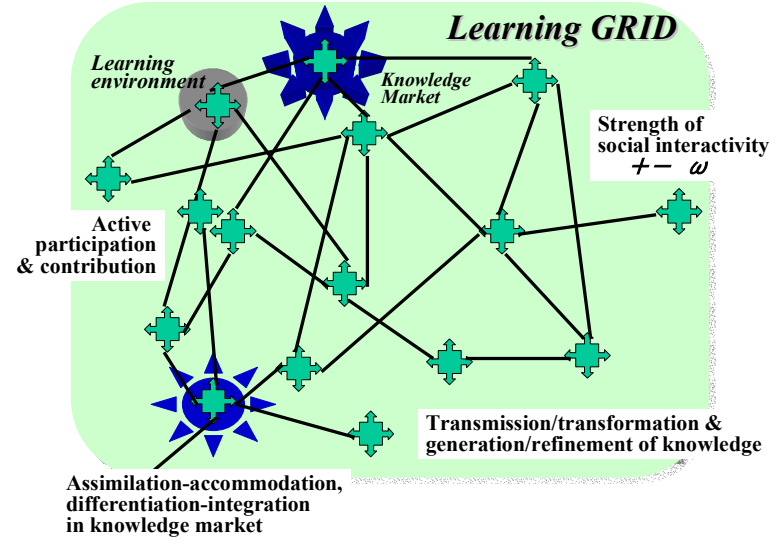

Figure 1: The Concept of Collaborative Leaming and Leaming GRID

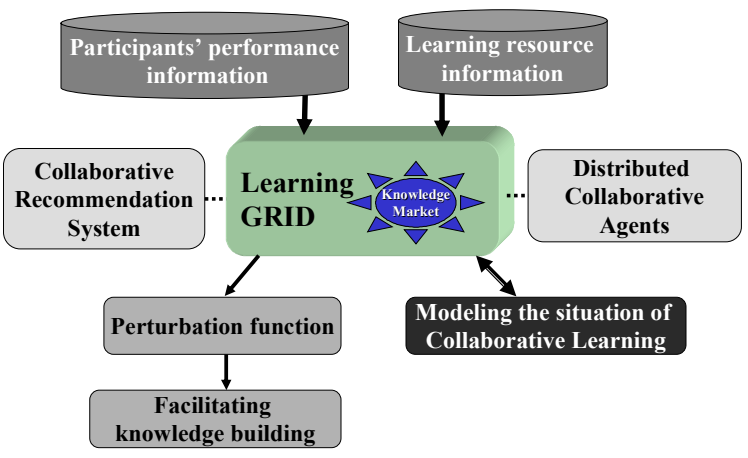

Figure 2: The Scheme andFunction of Leaming GRID Technology

acquiring by distributed collaborative agents in order to facilitate knowledge building.

Learning GRID provides adhesive fundamentals for social computing which has also the function of collaborative filtering with recommendation function. So, we can regard this as a conductor in a marketplace and a kind of negotiation-circulation engine for knowledge management. Every agent is embedded in every Learning GRID in Internet space, each agent tries to communicate, exchange need-information among Learning GRID. In some cases, plural agents behave collaboratively or competitively for taking well knowledge negotiation along with participants' requirement. Therefore, the agent's roles become to be quite important within/among Learning GRID. We set up two kinds of agent who are 1) Learning GRID agent (LGgent), 2) Knowledge Messenger agent(KMgent) for investigation, collaboration and whole-watching in the GRID world.

\section{Knowledge Management and Collaborative Memory in the GRID World}

\subsection{The Management of Learning Information}

The abstract mechanism of the management of learning information developed in this study is shown in figure 3 . The processing mechanism consists of two components. The first one is a module that offers the learning environment. The second one is the $\mathrm{CM}$ that controls various information and data produced from the learning environment.

In the learning environment, 2 types of functions are offered. One is the monitoring function for the learning progress. The other is the tool/application for the CL. The former function controls the learning history/record of individual learners and the progress of the collaborative group learning. The latter tool/application becomes a space/workplace for collaborative synchronous /asynchronous 


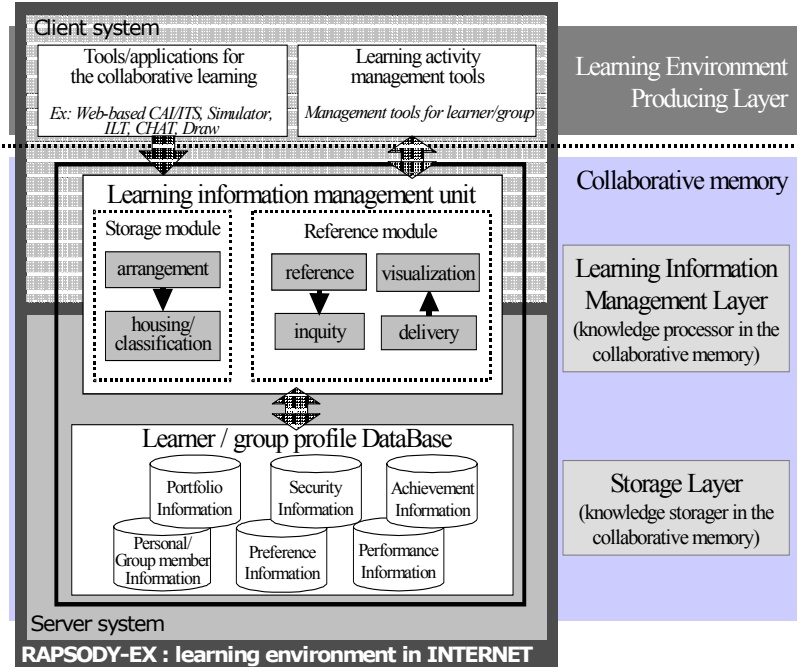

Figure 3: The Mechanism Scheme of the KnowledgeMarketplace (i.e. REX)

learning. The learning information, which emerged from such a learning environment, is handed to the $\mathrm{CM}$.

The CM offers 2 types of functions. One is the knowledge processing function, and the other is the knowledge storage function. In the former, input learning information is shaped to the defined form. In the latter, for the formatted information, some attributes related to content are added. The complex information processing takes place in the $\mathrm{CM}$.

\subsection{The Collaborative Memory}

The CM consists of two parts. One is a information and knowledge processing/management mechanism, which integrates and organizes the stored learning information and knowledge based on the purpose of usage and/or reference them. The other is a structured memory space, where diverse information and knowledge on learning are stored. In the CM, information generation / arrangement / housing / reference / visualization are the management processes of expressive knowledge in the learning space. REX is a learning environment, which possesses a knowledge management mechanism. This function works soundly with CM. In this environment, the following functions are realized in the learning space.

1)the review of the learning process,

2)the summarization of the problem solving process

3) the reference of other learners' problem solving method

Learning information is expressed by a unified format. Then, that information is accumulated in the CM. This information becomes the reference object of the learner. The generation and the management of the information on the learning performance and the portfolio of the learner and group are main objects of the knowledge management. In this study, learning information is obtained from the application tools for the CL. It is necessary to control the learning record, the reference log of the others' learning information and the log of problem solving and learning progress. To realize this control not only techniques based on symbolic knowledge processing approach, but also techniques based on sub-symbolic knowledge processing approach are used.

The CM consists of two layers. One is the information storage layer and the other one is the management layer of the stored information. At the information storage layer, 4 kinds of information are mainly processes.

1) Learning information,

2) Information on the learner,

3) Information on the setting of the learning environment

4) Information on the learning result.
At the information management layer, the reference/ arrangement/integration of learning information are processed. The individual learner profile information is composed of information following the IEEE Profile information guidelines (IEEE 2000). The group information is expressed by the expansion of the individual learner profile information. The conversion from the learning log data to learning information is necessary to develop this profile database. The information, which should apply in learning information, is as follows:

- Information and/or data on its learning context and/or learning situation

- Information about the sender and the sendee of the information

- Significance and/or outline in the educational context

- Information on the relation structure of the learning information

- Reference pointer to individual leamer and group who proposed or produced the information

- Relation with other material

\subsection{Pedagogical Agents in the GRID}

By adding above-mentioned information, the learning information is arranged into an unique form. If a learner requires some information related to his/her current learning, the learning market, like as a REX, shows the estimated/desired information to the learner. Figure 4 shows a communication scheme in the GRID. Two types of agent are in the GRID world. The learning GRID agent (LGgent) and the knowledge messenger agent (KMgent). These agents work for investigation, collaboration and whole-watching in the GRID world.

LGgent are organized by three types of agents. They are existed in the learning market. In the case of REX market, these agents perform each mission with user (user-RAPgent), each CL tool (application-RAPgent) and the CM in REX server (CM-RAPgent). Communication protocol between RAPgents is defined based on the FIPA ACL communicative act. The missions of each RAPgent are to transform information adaptively to create group portfolio, to maintain learning contexts in the group member and to let refer information in CM. To realize the knowledge management in REX, application-RAPgents develop some learning contexts by using learning information in the $\mathrm{CM}$. Then they refer the suitable learning information for the collaborative tool/application.

\section{The Learning GRID Technology in REX}

The knowledge management in educational context is defined as follows: "the systematic process of finding, selecting, organizing, distilling and presenting information in a way that improves a learner's comprehension and/or ability to fulfill hisher current learning objectives." The information of learning entity contains the expressed knowledge by learners. This overt knowledge can be represented by natural language as verbal information. So, we can regard this knowledge as one that would be elicited from the learner's tacit

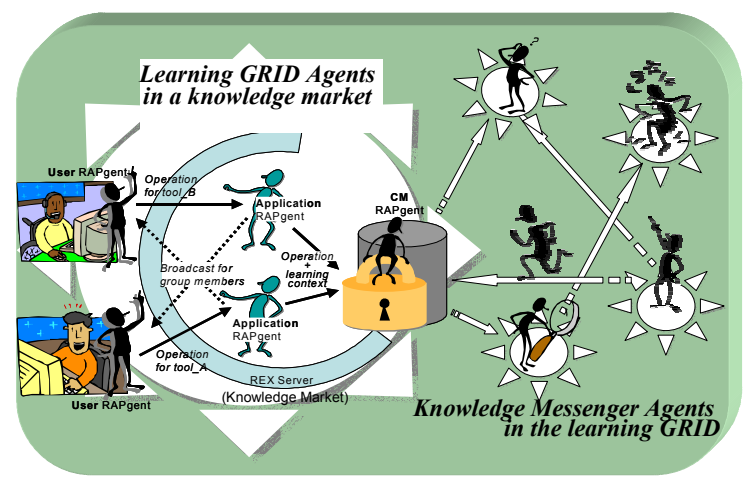

Figure 4: Agents Communication Scheme in the Leaming GRID 
knowledge. In this situation, what we have to consider is as follows:

- Who are the subjects of our knowledge managementwork?

Learners and the persons who support the learners are our subjects. Leamers' task is to acquire the ability/skill for the problem solving. On the other hand, supporters' tasks are to support for acquisition of ability/skill of the learner, and to support of the problem solving by the learner. Supporter means a facilitator/tutor/coach/ organizer etc.

- What are the knowledge resources in the learning group?

For learners, the knowledge for the effective and efficient problem solving is their knowledge resource. On the other hand, for the supporters, the knowledge on problem setting and activity assessment is their knowledge source.

- What is the gain for the learning group? The gains for learners are to acquire the ability in which to effectively and efficiently solve the problem, and to acquire the metacognition ability. For supporters, the acquisition of the ability of supporting the ability acquisition of the learner is their gain.

- How are the knowledge resources controlled to guarantee the maximum gain for the learning group?

By the information processing to relate common knowledge of the $\mathrm{CM}$ and learning context, we try to manage the knowledge in the CL. To create the collaborative portfolio between individual and group learning, extension of acquired knowledge of learners, knowledge extraction from learning history under the problem solving and making outline of problem solving process.

\section{REX as a Learning Market 6.1 Learning GRID with REX}

A learner group that guaranties the smooth transmission of knowledge can form a community (the knowledge community) by sharing and reusing common knowledge. The image of collaborative/ group learning with REX is shown in figure 5 Learning activities that occur within this group are as follows:

- Achievement of learning objectives as a group;

- Achievement of the learning objectives of each learner,

- Achievement of the learning objectives of the learner group, which consists of multiple learners.

REX supports the transmission of knowledge in the learner group and the promotion of the learning activity. It is indispensable that REX has the following functions:

1) Function which controls learning information for the individual learner and the group.

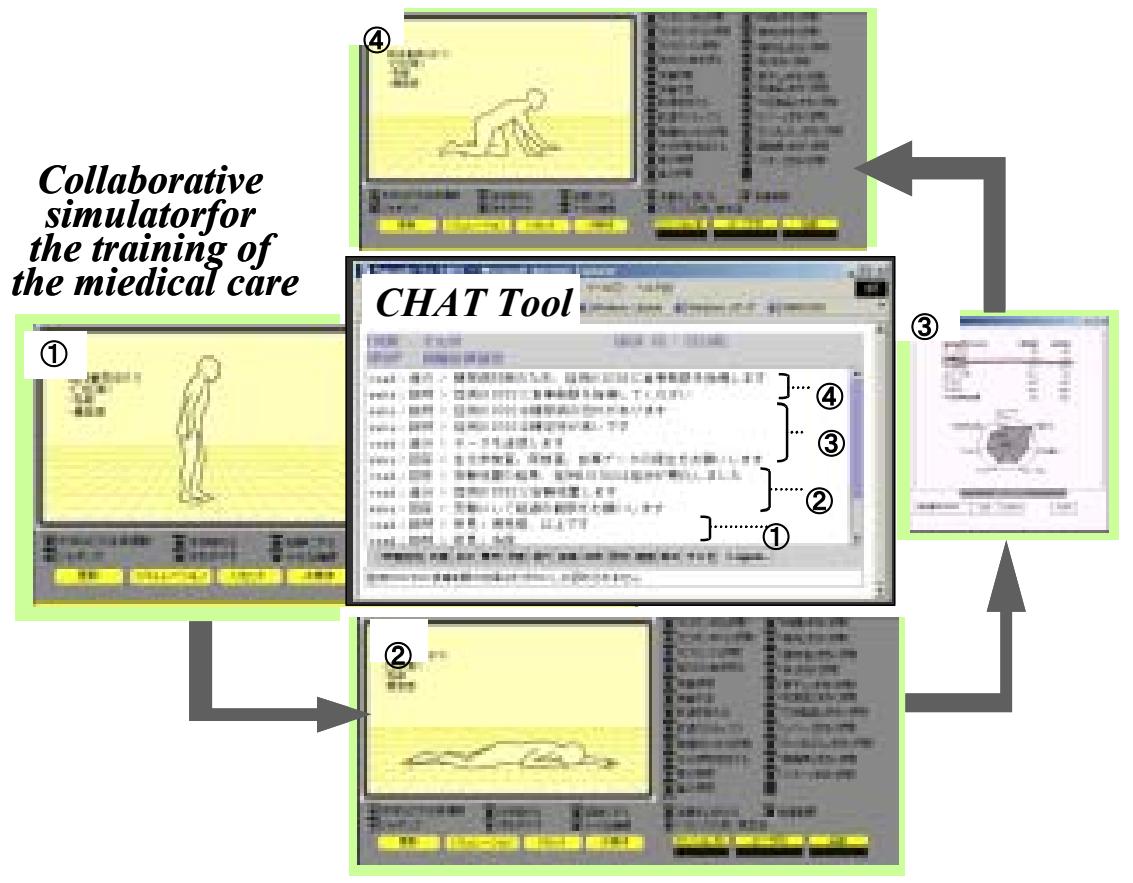

Figure 5: Collaborative Leaming on the REX with Two Types of Collaborative Applications $<$ atext chat tool and a collaborative simulator for the nutrition treatment $>$
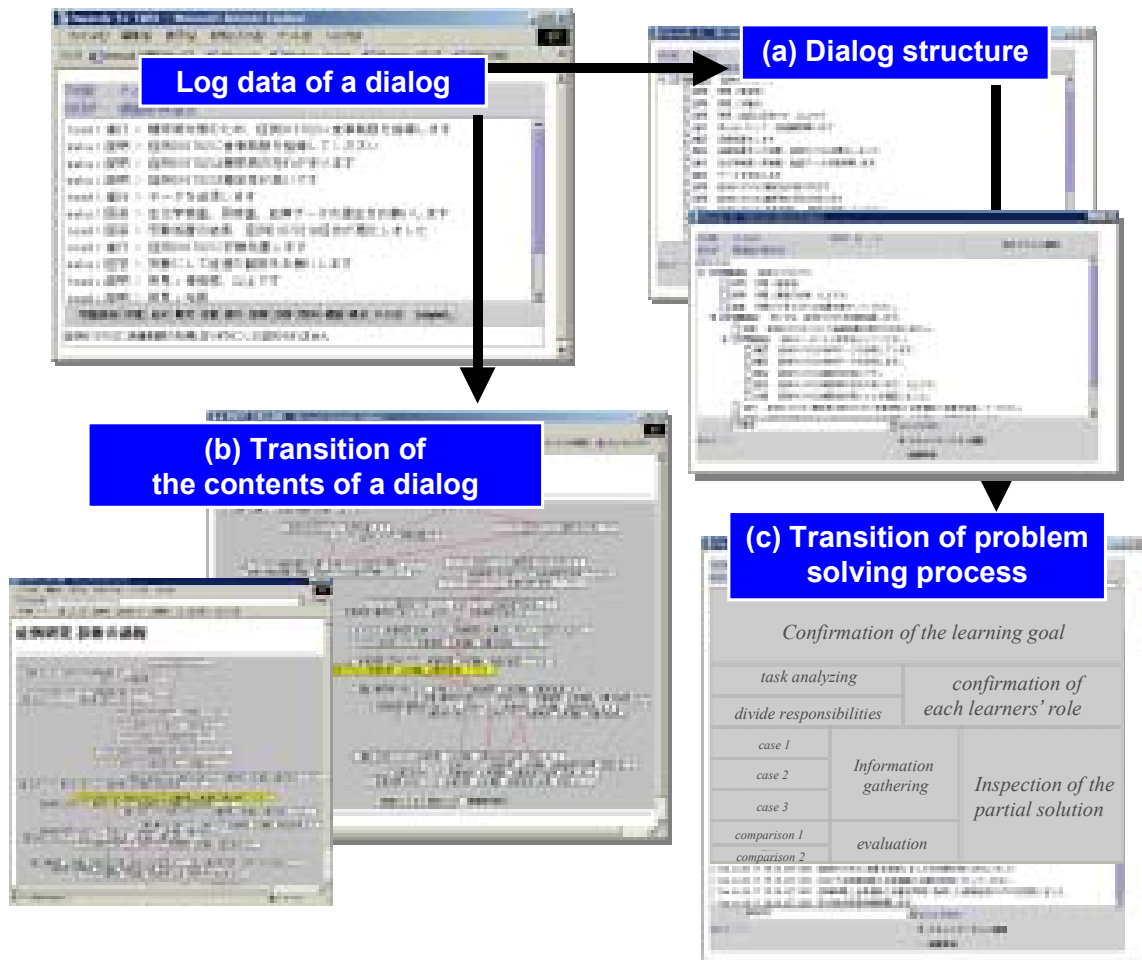

Figure 6:Examples of Leaming Knowledge Management in the Leaming GRID

2) Function, which manages learning information of the learner formediation.

The learner and group information are produced from the learning space. This information will be stored in the collaborative memory (CM). This information is defined as learning information. We also define the method of information management of such information and the structure of the $\mathrm{CM}$. 


\subsection{Examples of the Knowledge Management in REX}

Figure 6 shows the window images of the collaborative applications on REX. Two types of applications are loaded. One is a chat tool for the text communication among the group member. Another application is a collaborative simulator for the . Each application has each application-RAPgent. By the functions of these RAPgents, learning history data at this session is stored in the CM and formulates a set of the group portfolio.

The examples of knowledge management at this session are shown in the figure 6. A log data of this dialog is visualized by three kinds of methods. These results are produced by three application RAPgents. The first method is visualization of the dialog structure (figure 6 (a)). The dialog layers are reasoned based on the dialog proceeding model (Inaba 1997) and the utterance intention information that were given to the dialog log. The result is shown as tree structure. The second method is visualization of transition of the contents of a dialog (figure 6 (b)). An appearance of the important term that is in a dialog is searched for using the term dictionary about the current discussion/learning domain (Chiku 2001). This result and the timing connection of each utterance are considered to detect a transition of the contents of a dialog. The result is shown as graph structure. The third method is visualization of transition of problem solution process (figure 6 (c)). One utterance can be unified as meaningless unit for the problem solving process from the first and the second processing result and an educational mentor's expertise/educational intentions. The result re-constituted as problem solution process is shown with the structure that imitated the dendrogram.

\section{Evaluation and Conclusions}

Based on these concepts, we examined the feasibility, implementability and effectivity of our GRID world. The learning GRID was constructed by some collaborative applications (Collaborative simulator for the nutrition treatment / the company management/economical control ), some communication tools (text/audio/image) and the REX as a knowledge marketplace(Kayama 2002-a, Kayama 2002-b).

The purpose of this study is to support the learning activity in the Internet learning space. We examine the knowledge management and the knowledge representation of the learning information for the CL support. REX is a distributed learning support environment organized as a learning GRID technology.

In this paper, the management of learning information in REX is described. REX is an integrated distributed learning environment and the GRID technology for supporting tools/ applications for the CL. Also, in this paper, the knowledge management mechanism in the educational context is showed. The details of GRID technology as knowledge management and for connecting different soft wares is exploring by using the semantic web approach and it will be integrated with the current learning support environment.

Our Grid technology is focused in the center of connecting various information among learners' profile, log-data in learning process and learning resources as information Grid for the purpose of sharing with interoperability of applications/tools used in collaborative learning process.

\section{References}

ADLNet 2000. Shareble Courseware Object Reference Model:SCORM, Ver.1.0, http:/www.adlnetorg/.

Agent Society, http://www.agentorg/

A. Inaba and T. Okamoto 1997. Negotiation Process Model for intelligent discussion coordinating system on CSCL environment, Proceedings of the AIED97,pp. 175-182.
A. R. Kaye 1994. Computer Supported Collaborative leaming in a MultiMedia Distance Education Environment, in Claire OMalley (Ed.) ComputerSupported Collaborative leaming, pp.125-143, Springer-Verlag.

B. Collis 1999. Design, Development and Implementation of a WWW-Based Course-SupportSystem, Proceedings of ICCE99, pp.11-18.

FIPA, http:/drogo.cseltstet.itfipal

G. Cumming, T. Okamoto and L. Gomes 1998. Advanced Research in Computers in Education, IOS press.

H. Cowie and J. Ruddick, 1988 Co-operative Group Work; an overview, BP Educational Service, Sheffield University

J. Elliott 1993. What have we Leamed from Action Research in School-based Evaluation, Educational Action Research,Vol.1, No.1,pp175-186.

J. Roschelle and S. D. Teasley 1995 The construction of shared knowledge in collaborative problem solving, In C.E. OMalley (Ed.) ComputerSupported Collaborative learing, pp.69-97, Springer-Verlag.

IEEE 2000. Draft Standard for Leaming Technology -Public and Private Information(PAPI) for Leamer, IEEEP1484.2D6, hitp://tscieee.org/.

IMS 1998. Leaming Resourcde Metadata : Information Model, Best Practice and Implementation Guide, IMS Ver1.0, http:/www.imsproject.org/.

IPSJ Joumal 1999. Special Issue on Multimedia • Distributed and Cooperative Computing, Vol.39 No.02.

Joumal of Intemational Fonum of Educational Technology \& Society 2000. Special issue on Theme: On-line Collaborative leaming Environment, Vol.3,No.3.

Joumal of Interactive Leaming Research 1999. Special Issue on Intelligent Agents forEducational Computer-Aided Systems,Vol.10,Nos.3-4.

L. Colazzo, and A. Molinari 1996. Using Hypertext Projection to Increase Teaching Effectiveness, Intemational Joumal of Educational Multimedia and Hypermedia, AACE.

M. Chiku etal. 2001. "A dialog visualization tool : Gijiroku", Proceedings of the 62th Annual Conference of the Information Processing Sciety of Japan, pp.241-244.

M. Kayama and T. Okamoto, 2002, A Leaming Framework for the Internet Based Collaborative Leaming -sharing workplace and sharing leaming knowledge-, Proceedings of the 1st Fonum of Information Technology 2002,pp.313-314.

M. Kayama and T. Okamoto, 2002, Collaborative Technologies and a Leaming Framework for Collaborative Leaming, Proceedings of thel8th Annual Conference of the Japanese Society of Educational Technology, pp.149-152.

M. Kobayashi ei al. 1998. Collaborative Customer Services Using Synchronous Web Browser Sharing, Proceedings of CSCW 98, pp.99$108,1998$.

OMG,http//www.omg.org/

P. Dillenbourg, 1999 Collaborative leaming - Cognitive and Computational Approaches -, Pergamon.

S. McNeil etal 1998. Technology and Teacher Education Annual, AACE

T. Chan etal. 1997. Global Education ON the Net, Springer-Verlag.

T.Davenport 1997. Working Knowledge, Harvard Business School Press.

The United Kingdum Govemment 1997. Connecting the Leaming Society, The United Kingdum Govemment's Consultation paper.

T. Kuhn 1962. The structure of scientific revolutions, University of Chicago Press.

T. Okamoto, A.I.Cristea and M. Kayama 2000. Towards Intelligent MediaOriented Distance Leaming and Education Environments, Proceedings of ICCE2000

University of Phoenix Home Page: http://www. uophx.edu/

Jones Intemational University Home Page: $h$ ttp:/www.jonesintemational.edu/

V.Pankratius and G. Vossen. 2003. Towards e-Leaming Grids: Using Grid Computing in Electronic Leaming. Technical Report Nr. 98, Dept . of Information Systems, Universityof Muenster.

V. Pankratius and G. Vossen. 2003. Towards the Utilization of Grid Computing in e-Leaming in Jose C. Cunha and Omer F, Rana (Eds) "Grid Computing. Software Environments and Tools", Springer Verlag.

W.L. Johnson, and E. Shaw 1997. Using Agents to Overcome Deficiencies in Web-Based Courseware, Proceedings of the workshop "Intelligent Educational Systems on the World Wide Web" at the 8th World Conference of the AIED Society, IV-2.

Y. Aoki and A. Nakajima 1999. User-Side Web Page Customization, Proceedings of 8th Intemational Conference on HCI, Vol.1,pp.580-584.

Y. Shimizu 1999., Toward a New Distance Education System, Proceedings ofICCE99,pp.69-75. 